\title{
A Unified Numerical Framework Model for Simulating Flow, Transport, and Heat Transfer in Porous and Fractured Media
}

\author{
Yu-Shu Wu \\ Earth Sciences Division, Lawrence Berkeley National Laboratory, One Cyclotron Road, \\ Berkeley, CA 94720, USA
}

\begin{abstract}
It has long been recognized that a common ground exists between governing equations used for describing various flow and transport phenomena in porous media. Put another way they are all generally based on the same form of mass and/or energy conservation laws. This implies that there may exist a unified formulation and numerical scheme applicable to modeling all of these physical processes. This paper explores such a possibility and proposes a generalized framework, as well as a mathematical formulation for modeling all known transport phenomena in porous media. Based on this framework, a unified numerical approach is developed and tested using multidimensional, multiphase flow, isothermal and nonisothermal reservoir simulators. In this approach, a spatial domain of interest is discretized with an unstructured grid, then a time discretization is carried out with a backward, first-order, finite-difference method. The final discrete nonlinear equations are handled fully implicitly, using Newton iteration. In addition, the fracture medium is handled using a general dualcontinuum concept with continuum or discrete modeling methods. A number of applications are discussed to demonstrate that with this unified approach, modeling a particular porousmedium flow and transport process simply becomes a matter of defining a set of state variables, along with their interrelations or mutual influence.
\end{abstract}

\section{INTRODUCTION}

Since the late 1950s, significant progress has been made in developing and applying numerical simulation techniques in petroleum engineering $[3,1,12,17]$ and in groundwater literature $[9,10]$. Because of its generality and effectiveness in handling subsurface multiphase flow and transport problems, the numerical simulation technique has become the major tool used by scientists and engineers in studies of flow and transport processes within a porous medium. Numerical modeling approaches currently used for simulating multiphase flow and transport processes are generally based on methodologies developed for petroleum and geothermal reservoir simulations, as well as groundwater modeling. They involve solving fully coupled formulations describing these processes, using finite-difference or finite-element schemes with a volume-averaging approach.

Continual research effort, driven by the need to develop underground natural resources and resolve subsurface contamination problems, has developed and provided many numerical modeling approaches and models for field applications. Mathematical modeling techniques 
have become very sophisticated and are capable of routinely solving problems ranging from simple groundwater flow to coupled multiple physical process simulations. It has long been recognized that a common ground exists between governing equations used for describing various flow and transport phenomena in porous media (i.e., they are all generally based on the same form of mass and/or energy conservation laws). This suggests that there may exist a unified formulation and numerical scheme applicable to modeling all of these physical processes. To explore such a possibility, this paper proposes a generalized framework as well as mathematical formulation for modeling all known transport phenomena in porous media. Based on this framework, a unified numerical approach is developed for simulating singlephase and multiphase flow, multicomponent chemical transport, and heat transfer in porous and fractured reservoirs.

The proposed unified numerical approach is tested with multidimensional, multiphaseflow, nonisothermal reservoir simulators. In this approach, a subsurface domain is discretized using an unstructured grid, followed by time discretization carried out using a backward, firstorder, finite-difference method. The final discrete linear or nonlinear equations are handled fully implicitly, using Newton iteration. In addition, the fracture medium is handled using a general dual-continuum concept with continuum or discrete modeling approaches. We demonstrate that with this unified approach, modeling a particular process of porous-medium flow and transport becomes simply a matter of defining a set of state variables, along with their interrelations or mutual effects.

\section{GENERALIZED GOVERNING EQUATIONS}

The physical processes associated with flow and transport in porous media are governed by the same fundamental conservation laws as those used in other branches of the sciences and engineering: conservation of mass, momentum, and energy governs the behavior of fluid flow, chemical transport, and heat transfer in rock. These physical laws are often represented mathematically on the macroscopic level by a set of partial differential or integral equations, called governing equations. These governing equations are generally nonlinear as long as compressible or multiphase fluids or heat transfer is involved and needed to quantitatively model the flow and transport processes occurring in porous media. Based on the general conservation laws, we present a set of generalized governing equations for multiphase fluid flow, multicomponent transport, and heat transfer in porous and fractured media, providing a framework for numerical formulations to cover all possible scenarios for flow and transport in porous media.

Let us consider a multiphase, nonisothermal system consisting of several fluid phases, such as gas, water, and oil (NAPL), with each fluid phase in turn consisting of a number of mass components. To derive a set of generalized governing equations for multiphase fluid flow, multicomponent transport, and heat transfer, we assume that these processes can be described using a continuum approach within a representative elementary volume (REV) in a porous or fractured medium [2]. In addition, a condition of local thermodynamic equilibrium is assumed so that at any time temperatures, phase pressures, densities, viscosities, enthalpies, internal energies, and component concentrations (or mass fractions) are the same locally at each REV of the porous medium. 
According to mass and energy conservation principles, a generalized conservation equation of mass components and energy in the porous continuum can be written as follows:

$\frac{\partial \mathrm{M}^{\mathrm{k}}}{\partial \mathrm{t}}=\mathrm{G}^{\mathrm{k}}+\mathrm{q}^{\mathrm{k}}+\mathrm{F}^{\mathrm{k}}$

where superscript $\mathrm{k}$ is the index for the components, $\mathrm{k}=1,2,3, \ldots, \mathrm{N}_{\mathrm{c}}$, with $\mathrm{N}_{\mathrm{c}}$ being the total number of mass components and with $\mathrm{k}=\mathrm{N}_{\mathrm{c}}+1$ for energy "component" (note that heat energy is regarded as a component for convenience); $\mathrm{M}$ is the accumulation term of component $\mathrm{k} ; \mathrm{G}^{\mathrm{k}}$ is the decay or internal generation (reaction) term of mass or energy components; $\mathrm{q}^{\mathrm{k}}$ is an external source/sink term or fracture-matrix exchange term for mass or energy component $\mathrm{k}$ and energy; and $\mathrm{F}^{\mathrm{k}}$ is the "flow" term of mass or energy movement or net exchange from single-phase and multiphase flow, or diffusive and dispersive mass transport, or heat transfer, as discussed below.

In addition to the conservation or continuity equations of mass and thermal energy, shown in Equation (1), we also need specific relationships or mechanisms that describe why and how fluid flow, solute transport, and heat transfer occur in porous and fractured media. This is to define the "flow" term in Equation (1), and the following specific laws act as such mechanisms by governing local fluid flow, component transport, and heat transfer processes in porous media.

\subsection{Single-Phase and Multiphase Flow}

For single-phase liquid or gas flow, Richards' equation, two active or three phase flow, if these fluids are treated as immiscible or mass exchange between phases can be ignored, the accumulation terms in (1) for gas (air), water and/or oil (NAPL) components in are evaluated as

$\mathrm{M}^{\beta}=\sum_{\beta}\left(\phi \rho_{\beta} \mathrm{S}_{\beta}\right)$

where superscript and subscript $\beta$ is an index for fluid phase $(\beta=\mathrm{g}$ for gas, $=\mathrm{w}$ for aqueous phase, $=0$ for oil); $\phi$ is the porosity of porous media; $\rho_{\beta}$ is the density of phase $\beta$; and $S_{\beta}$ is the saturation of phase $\beta$. Note that in this special case, component $k$ (by superscript) and phase (by subscript) are interchangeable.

In this case, the decay or generation term is negligible with

$\mathrm{G}^{\beta}=0$

The mass flow term is determined by

$$
\mathrm{F}^{\beta}=\nabla \cdot\left(\rho_{\beta} \mathbf{v}_{\beta}\right)
$$


where $\mathbf{v}_{\beta}$ is a vector of the Darcy's velocity or volumetric flow, defined by Darcy's law to describe the flow of single or multiple immiscible fluids [15], as:

$$
\mathbf{v}_{\beta}=-\frac{\mathrm{kk}_{\mathrm{r} \beta}}{\mu_{\beta}}\left(\nabla \mathrm{P}_{\beta}-\rho_{\beta} \mathrm{g} \nabla \mathrm{z}\right)
$$

where $\mathrm{P}_{\beta}, \mu_{\beta}$, and $\mathrm{g}$ are pressure, viscosity of fluid phase $\beta$, and gravitational constant, respectively; $\mathrm{z}$ is the vertical coordinate; $\mathrm{k}$ is absolute or intrinsic permeability (a tensor in general); and $\mathrm{k}_{\mathrm{r} \beta}$ is the relative permeability to phase $\beta$ (equal to one for single-phase flow, i.e., single-phase is considered as a special case of multiphase flow).

\subsection{Mass Transport}

The movement of dissolved mass components or chemical species in a multiphase porous medium system can also be handled as a special case of Equation (1). The accumulation terms for component $\mathrm{k}$ is

$$
\mathrm{M}^{\mathrm{k}}=\phi \sum_{\beta}\left(\rho_{\beta} \mathrm{S}_{\beta} \mathrm{X}_{\beta}^{\mathrm{k}}\right)+(1-\phi) \rho_{\mathrm{s}} \rho_{\mathrm{w}} \mathrm{X}_{\mathrm{w}}^{\mathrm{k}} \mathrm{K}_{\mathrm{d}}^{\mathrm{k}} \quad\left(\mathrm{k}=1,2,3, \ldots, \mathrm{N}_{\mathrm{c}}\right)
$$

where $\mathrm{X}_{\beta}^{\mathrm{k}}$ is the mass fraction of component $\mathrm{k}$ in fluid $\beta ; \rho_{\mathrm{s}}$ is the density of rock solids; and $\mathrm{K}_{\mathrm{d}}^{\mathrm{k}}$ is the distribution coefficient of component $\mathrm{k}$ between the aqueous phase and rock solids to account for adsorption effects.

In the case in which components are subject to a first-order radioactive decay, the decay/generation term is

$$
\mathrm{G}^{\mathrm{k}}=\phi \lambda_{\mathrm{k}}\left(\sum_{\beta}\left(\rho_{\beta} \mathrm{S}_{\beta} \mathrm{X}_{\beta}^{\mathrm{k}}\right)+(1-\phi) \rho_{\mathrm{s}} \rho_{\mathrm{w}} \mathrm{X}_{\mathrm{w}}^{\mathrm{k}} \mathrm{K}_{\mathrm{d}}^{\mathrm{k}}\right) \quad\left(\mathrm{k}=1,2,3, \ldots, \mathrm{N}_{\mathrm{c}}\right)
$$

where $\lambda_{\mathrm{k}}$ is the radioactive decay constant of component $\mathrm{k}$.

The mass component transport is governed in general by processes of advection, diffusion, and dispersion, and is also subject to other processes such as radioactive decay, adsorption, dissolution and precipitation, mass exchange and partition between phases, or chemical reactions. Advective transport of a component or solute is carried by flow of a fluid, and diffusive and dispersive flux is contributed by molecular diffusion and mechanical dispersion, or hydrodynamic dispersion. These processes are described using a modified Fick's law for

transport through a single-phase porous medium $[16,2]$. Then, the total mass flow term for a component $\mathrm{k}$, by advection and dispersion, is written as

$$
\mathrm{F}^{\mathrm{k}}=-\sum_{\beta} \nabla \cdot\left(\rho_{\beta} \mathrm{X}_{\beta}^{\mathrm{k}} \mathbf{v}_{\beta}\right)+\sum_{\beta} \nabla \cdot\left(\underline{\mathrm{D}}_{\beta}^{\mathrm{k}} \bullet \nabla\left(\rho_{\beta} \mathrm{X}_{\beta}^{\mathrm{k}}\right)\right) \quad\left(\mathrm{k}=1,2,3, \ldots, \mathrm{N}_{\mathrm{c}}\right)
$$


This indicates that the mass flow consists of two parts, the first part, i.e., the first term on the left-hand side of Equation (8), is contributed by advection in all phasesand the second part (the second term on the left-hand side of (8)) is diffusive flux by hydrodynamic dispersion. In (8), $\underline{\mathrm{D}}_{\beta}^{\mathrm{k}}$ is the hydrodynamic dispersion tensor accounting for both molecular diffusion and mechanical dispersion for component $\mathrm{k}$ in phase $\beta$, defined by an extended dispersion model [16] to include multiphase effects as,

$\underline{D}_{\beta}^{\mathrm{k}}=\alpha_{\mathrm{T}}^{\beta}\left|\mathbf{v}_{\beta}\right| \delta_{\mathrm{ij}}+\left(\alpha_{\mathrm{L}}^{\beta}-\alpha_{\mathrm{T}}^{\beta}\right) \frac{\mathbf{v}_{\beta} \mathbf{v}_{\beta}}{\left|\mathbf{v}_{\beta}\right|}+\phi \mathrm{S}_{\beta} \tau \mathrm{d}_{\beta}^{\mathrm{k}} \delta_{\mathrm{ij}} \quad\left(\mathrm{k}=1,2,3, \ldots, \mathrm{N}_{\mathrm{c}}\right)$

where $\alpha_{\mathrm{T}}^{\beta}$ and $\alpha_{\mathrm{L}}^{\beta}$ are transverse and longitudinal dispersivities, respectively, in fluid $\beta$ of porous media; $\tau$ is tortuosity of the porous medium; $d_{\beta}^{\mathrm{k}}$ is the molecular diffusion coefficient of component $\mathrm{k}$ within fluid $\beta$; and $\delta_{\mathrm{ij}}$ is the Kronecker delta function $\left(\delta_{\mathrm{ij}}=1\right.$ for $\mathrm{i}=\mathrm{j}$, and $\delta_{\mathrm{ij}}$ $=0$ for $\mathrm{i} \neq \mathrm{j}$ ), with $\mathrm{i}$ and $\mathrm{j}$ being coordinate indices.

\subsection{Heat Transfer}

The accumulation term for the heat equation is usually is defined as

$\mathrm{M}^{\mathrm{N}_{\mathrm{c}}+1}=\sum_{\beta}\left(\phi \rho_{\beta} \mathrm{S}_{\beta} \mathrm{U}_{\beta}\right)+(1-\phi) \rho_{\mathrm{s}} \mathrm{U}_{\mathrm{s}}$

where $\rho_{\mathrm{s}}$ is the density of rock solids; and $U_{\beta}$ and $U_{s}$ are the internal energies of fluid $\beta$ and rock solids, respectively.

Heat transfer in porous media is in general a result of both convective and conductive processes, although in certain cases, radiation may also be involved. These heat-transfer processes are complicated by interactions between multiphase fluids, multicomponents, and associated changes in phases, internal energy, and enthalpy. Heat convection is contributed by thermal energy carried mainly by bulk flow of all fluids as well as by dispersive mass fluxes. On the other hand, heat conduction or radiation is driven by temperature gradients and may follow Fourier's law or Stefan-Boltzmann's law, respectively. Then the combined, overall heat flux term, owe to convection, conduction and radiation in a multiphase, multicomponent, porous medium system, may be described as

$$
\mathrm{F}^{\mathrm{N}_{\mathrm{c}}+1}=-\sum_{\beta} \nabla \bullet\left(\mathrm{h}_{\beta} \rho_{\beta} \mathbf{v}_{\beta}\right)+\sum_{\beta} \sum_{\mathrm{k}} \nabla \bullet\left(\mathrm{h}_{\beta}^{\mathrm{k}} \underline{\mathrm{D}}_{\beta}^{\mathrm{k}} \bullet \nabla\left(\rho_{\beta} \mathrm{X}_{\beta}^{\mathrm{k}}\right)\right)+\nabla \bullet\left(\mathrm{K}_{\mathrm{T}} \nabla \mathrm{T}\right)-\varepsilon \sigma_{0} \mathrm{~T}^{4}
$$

where $h_{\beta}$ and $h_{\beta}^{k}$ are specific enthalpies of fluid phase $\beta$ and of component $\mathrm{k}$ in fluid $\beta$, respectively; $\mathrm{K}_{\mathrm{T}}$ is the overall thermal conductivity; $\mathrm{T}$ is temperature; $\varepsilon$ is a radiation emissivity factor, and $\sigma_{\mathrm{o}}\left(=5.6687 \times 10^{-8} \mathrm{~J} / \mathrm{m}^{2} \mathrm{~K}^{4}\right)$ is the Stefan-Boltzmann constant.

As shown in Equation (11), the total heat flow in a multiphase, multicomponent system is determined by heat convection of flow and mass dispersion (the first two terms on the right- 
hand side of (11)), heat conduction (the third term on the right-hand side), and thermal radiation (the last term on the right-hand side).

\subsection{Constitutive Relationships}

To complete the mathematical description of multiphase flow, multicomponent transport, and heat transfer in porous media, Equation (1), a generalized mass- and energy-balance equation, needs to be supplemented with a number of constitutive equations. These constitutive correlations express interrelationships and constraints of physical processes, variables, and parameters, and allow the evaluation of secondary variables and parameters as functions of a set of primary unknowns or variables selected to make the governing equations solvable. Table 1 lists a commonly used set of constitutive relationships for describing multiphase flow, multicomponent mass transport, and heat transfer through porous media. Many of these correlations for estimating properties and interrelationships are determined by experimental studies. 
Table 1

Constitutive Relationships and Functional Dependence

\begin{tabular}{|c|c|c|}
\hline Definition & Function & Description \\
\hline Fluid saturation & $\sum_{\beta} S_{\beta}=1$ & Constraint on summation of total fluid saturation. \\
\hline Mass fraction & & Constraint on mass fractions within phase $\beta$. \\
\hline Capillary pressure & $\mathrm{P}_{\mathrm{C} \beta}=\mathrm{P}_{\mathrm{C} \beta}\left(\mathrm{S}_{\beta}\right)$ & $\begin{array}{l}\text { In a multiphase system, the capillary pressure relates pressures } \\
\text { between the phases and is defined as functions of fluid } \\
\text { saturation. }\end{array}$ \\
\hline Relative permeability & $\mathrm{k}_{\mathrm{r} \beta}=\mathrm{k}_{\mathrm{r} \beta}\left(\mathrm{S}_{\beta}\right)$ & $\begin{array}{l}\text { The relative permeability of a fluid phase in a multiphase system } \\
\text { are normally assumed to be functions of fluid saturation. }\end{array}$ \\
\hline Fluid density & $\rho_{\beta}=\rho_{\beta}\left(\mathrm{P}, \mathrm{T}, \mathrm{X}_{\beta}^{\mathrm{k}}\right)$ & $\begin{array}{l}\text { Density of a fluid phase is treated as a function of pressure and } \\
\text { temperature, as well as mass compositions }\left(\mathrm{k}=1,2,3, \ldots, \mathrm{N}_{\mathrm{c}}\right) \text {. }\end{array}$ \\
\hline Fluid viscosity & $\mu_{\beta}=\mu_{\beta}\left(\mathrm{P}, \mathrm{T}, \mathrm{X}_{\beta}^{\mathrm{k}}\right)$ & $\begin{array}{l}\text { The functional dependence or empirical expressions of } \\
\text { viscosity of a fluid is treated as a function of pressure, } \\
\text { temperature, and composition. }\end{array}$ \\
\hline Henry's law & $\mathrm{P}_{\mathrm{g}}^{\mathrm{k}}=\mathrm{K}_{\mathrm{H}}^{\mathrm{k}} \omega_{\mathrm{w}}^{\mathrm{k}}$ & $\mathrm{P}_{\mathrm{g}}^{\mathrm{k}}$ is partial pressure of component $\mathrm{k}$ in gas phase; $\mathrm{K}_{\mathrm{H}}^{\mathrm{k}}$ is \\
\hline & & $\begin{array}{l}\text { Henry's constant for component } \mathrm{k} \text {; and } \omega_{\mathrm{w}}^{\mathrm{k}} \text { is the mole } \\
\text { fraction of component } \mathrm{k} \text { in water phase }\end{array}$ \\
\hline Equilibrium partitioning & $\omega_{\alpha}^{\mathrm{k}}=\mathrm{K}_{\alpha: \beta}^{\mathrm{k}} \omega_{\beta}^{\mathrm{k}}$ & $\omega_{\alpha}^{\mathrm{k}}$ and $\omega_{\beta}^{\mathrm{k}}$ are the mole fraction of component $\mathrm{k}$ in phase $\alpha$ \\
\hline & & and $\beta$, respectively; and $\mathrm{K}_{\alpha: \beta}^{\mathrm{k}}$ is the equilibrium partitioning \\
\hline Partitioning coefficient & $\mathrm{K}_{\alpha: \beta}^{\mathrm{k}}=\mathrm{K}_{\alpha: \beta}^{\mathrm{k}}\left(\mathrm{P}_{\beta}, \mathrm{T}, \mathrm{X}_{\beta}^{\mathrm{k}}\right)$ & $\begin{array}{l}\text { coefficient of component } \mathrm{k} \text { between phases } \alpha \text { and } \beta \text {. } \\
\text { depends on chemical properties of the component and is a } \\
\text { function of temperature, pressure and composition }\end{array}$ \\
\hline specific enthalpy of liquid & $\mathrm{h}_{\beta}=\mathrm{U}_{\beta}+\frac{\mathrm{P}_{\beta}}{\rho_{\beta}}$ & $\begin{array}{l}\text { Internal energy, } U_{\beta} \text {, of liquid phase } \beta \text { is a function of pressure } \\
\text { and temperature }\end{array}$ \\
\hline specific enthalpies of gas & $\mathrm{h}_{\mathrm{g}}^{\mathrm{k}}=\mathrm{U}_{\mathrm{g}}^{\mathrm{k}}+\frac{\mathrm{P}_{\mathrm{g}}^{\mathrm{k}}}{\mathrm{C}_{\mathrm{g}}^{\mathrm{k}}}$ & $\begin{array}{l}\mathrm{U}_{\mathrm{g}}^{\mathrm{k}} \text { the specific internal energy of component } \mathrm{k} \text { in the gas } \\
\text { phase; } \mathrm{C}_{\mathrm{g}}^{\mathrm{k}} \text { concentration of component } \mathrm{k} \text { in gas phase } \\
\left(\mathrm{kg} / \mathrm{m}^{3}\right)\end{array}$ \\
\hline thermal conductivity & $\mathrm{K}_{\mathrm{T}}=\mathrm{K}_{\mathrm{T}}\left(\mathrm{S}_{\beta}\right)$ & $\begin{array}{l}\text { The thermal conductivity of the porous medium is treated as a } \\
\text { function of fluid saturation. }\end{array}$ \\
\hline Porosity & $\phi=\phi^{\mathrm{o}}\left(1+\mathrm{C}_{\mathrm{r}}\left(\mathrm{P}-\mathrm{P}^{\mathrm{o}}\right)-\mathrm{C}_{\mathrm{T}}\left(\mathrm{T}-\mathrm{T}^{\mathrm{o}}\right)\right)$ & $\begin{array}{l}\phi^{\circ} \text { is the effective porosity at a reference pressure, } \mathrm{P}^{\mathrm{o}} \text {, and a } \\
\text { reference temperature, } \mathrm{T}^{\mathrm{o}} \text {; and } \mathrm{C}_{\mathrm{r}} \text { and } \mathrm{C}_{\mathrm{T}} \text { are the } \\
\text { compressibility and thermal expansion coefficient of the } \\
\text { medium, respectively }\end{array}$ \\
\hline Equilibrium adsorption & $\mathrm{X}_{\mathrm{s}}^{\mathrm{k}}=\mathrm{K}_{\mathrm{d}}^{\mathrm{k}} \rho_{\beta} \mathrm{X}_{\beta}^{\mathrm{k}}$ & $\begin{array}{l}\mathrm{X}_{\mathrm{s}}^{\mathrm{k}} \text { is the mass of component } \mathrm{k} \text { sorbed per mass of solids; and } \\
\text { the distribution coefficient, } \mathrm{K}_{\mathrm{d}}^{\mathrm{k}} \text {, is treated as a constant or as a } \\
\text { function of the concentration or mass fraction in a fluid phase } \\
\text { under the local chemical equilibrium condition }\end{array}$ \\
\hline Radioactive decay & $\mathrm{C}_{\beta}^{\mathrm{k}}=\mathrm{C}_{\beta 0}^{\mathrm{k}} \mathrm{e}^{-\lambda_{\mathrm{k}} \mathrm{t}}$ & $\begin{array}{l}C_{\beta}^{k} \text { is the concentration of component } \mathrm{k} \text { in phase } \beta \text { and is } \\
\text { equal to } C_{\beta 0}^{\mathrm{k}} \text { at } \mathrm{t}=0 ; \lambda_{\mathrm{k}} \text { is the radioactive decay constant }\end{array}$ \\
\hline First-order decay constant & $\lambda_{\mathrm{k}}=\frac{\ln (2)}{\mathrm{T}_{1 / 2}}$ & $\mathrm{~T}_{1 / 2}$ is the half-life of the radioactive component. \\
\hline
\end{tabular}




\section{NUMERICAL FORMULATION}

The methodology for using numerical approaches to simulate multiphase subsurface flow and transport consists in general of the following three steps: (1) spatial discretization of mass and energy conservation equations, (2) time discretization; and (3) iterative approaches to solve the resulting nonlinear, discrete algebraic equations. Among various numerical techniques for simulation studies, a mass- and energy-conserving discretization scheme, based on finite or integral finite-difference or finite-element methods, is the most commonly used approach, and is discussed here.

\subsection{Discrete Equations}

The component mass- and energy-balance Equation (1) are discretized in space using a control-volume concept. The control-volume approach provides a general spatial discretization scheme that can represent a one-, two- or three-dimensional domain using a set of discrete meshes. Each mesh has a certain control volume for a proper averaging or interpolation of flow and transport properties or thermodynamic variables. The control volume concept includes the conventional finite-difference scheme [12], an integral finitedifference method (Figure 1) [11, 13], a control-volume finite element [7], and Galerkin finite-element methods [8]. These are the most widely used discretization schemes for multiphase flow simulation. Time discretization is carried out using a backward, first-order, fully implicit finite-difference scheme. The discrete nonlinear equations for components of water, gas and oil, and heat at gridblock or node i can be written in a general form:

$$
\begin{aligned}
& \left\{A_{i}^{k, n+1}+G_{i}^{k, n+1} \Delta t-A_{i}^{k, n}\right\} \frac{V_{i}}{\Delta t}=\sum_{j \in \eta_{i}} \text { flow }_{i j}^{k, n+1}+Q_{i}^{k, n+1} \\
& \left(k=1,2,3, \ldots, N_{c}, N_{c}+1\right) \text { and }(i=1,2,3, \ldots, N)
\end{aligned}
$$

where superscript $\mathrm{k}$ serves also as an equation index for all mass components with $\mathrm{k}=1,2,3$, $\ldots, \mathrm{N}_{\mathrm{c}}$ and $\mathrm{k}=\mathrm{N}_{\mathrm{c}}+1$ denoting the heat equation; superscript $\mathrm{n}$ denotes the previous time level, with $\mathrm{n}+1$ the current time level to be solved; subscript $\mathrm{i}$ refers to the index of gridblock or node $\mathrm{I}$, with $\mathrm{N}$ being the total number of nodes in the grid; $\Delta \mathrm{t}$ is time step size; $\mathrm{V}_{\mathrm{i}}$ is the volume of node $i ; \eta_{i}$ contains the set of direct neighboring nodes $(j)$ of node $i ; A_{i}^{k}, G_{i}^{k}$, flow $_{\mathrm{ij}}^{\mathrm{k}}$, and $\mathrm{Q}_{\mathrm{i}}^{\mathrm{k}}$ are the accumulation and decay/generation terms, respectively, at node $\mathrm{i}$; the "flow" term between nodes $\mathrm{i}$ and $\mathrm{j}$, and sink/source term at node $\mathrm{i}$ for component $\mathrm{k}$ or thermal energy, respectively, are defined below. 

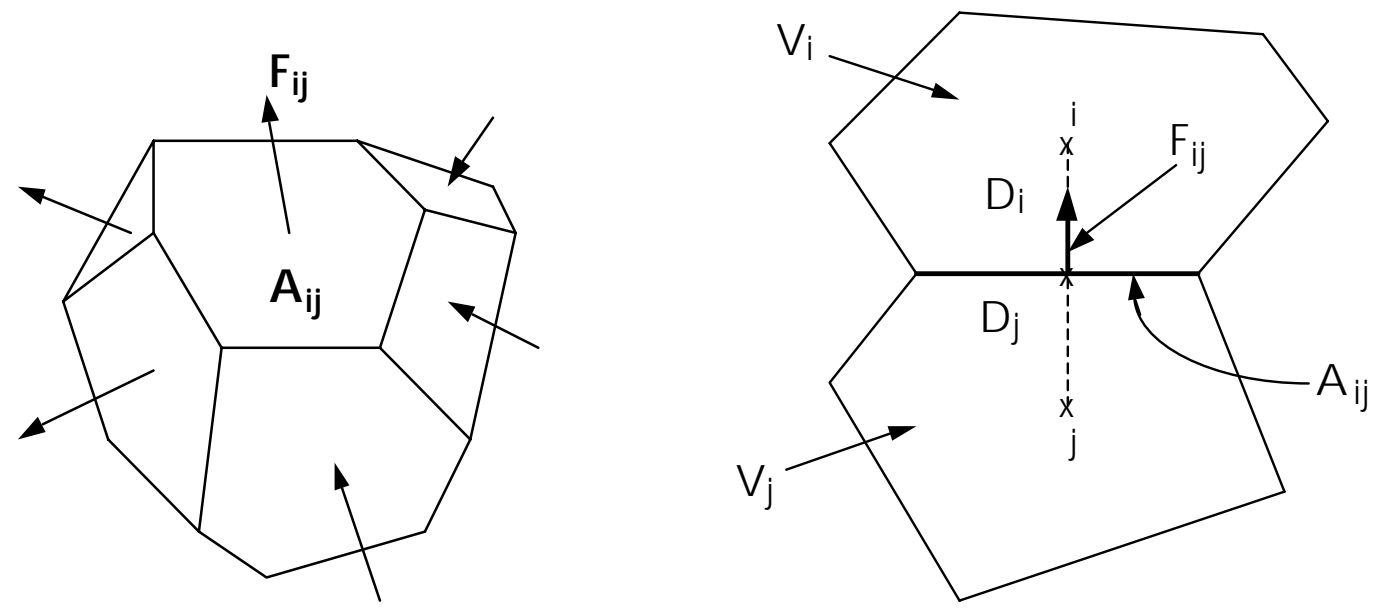

Figure 1. Space discretization and flow-term evaluation in the integral finite difference method (Pruess, 1991)

Equation (12) has the same form regardless of the dimensionality of the system, i.e., it applies to one-, two-, or three-dimensional flow, transport, and heat-transfer analyses. The accumulation and decay/generation terms for mass components or thermal energy are evaluated using Equations (6), (7), and (11), respectively, at each node i.

The "flow" terms in Equation (12) are generic and include mass fluxes by advective and dispersive processes, as described by Equation (4) or (8), as well as heat transfer, described by Equation (11). The mass flow term of Equation (12) for single-phase, Richards' or multiphase flow is described by a discrete version of Darcy's law, i.e., the mass flux of fluid phase $\beta$ along the connection is given by

flow $_{\mathrm{ij}}^{\beta}=\left(\frac{\rho_{\beta} \mathrm{k}_{\mathrm{r} \beta}}{\mu_{\beta}}\right)_{\mathrm{ij}+1 / 2} \gamma_{\mathrm{ij}}\left[\psi_{\beta \mathrm{j}}-\psi_{\beta \mathrm{i}}\right]$

where $\gamma_{\mathrm{ij}}$ is transmissivity and is defined differently for finite-difference or finite-element discretization. If the integral finite-difference scheme [13] is used, the transmissivity is calculated as

$\gamma_{i j}=\frac{A_{i j} k_{i j+1 / 2}}{D_{i}+D_{j}}$

where $A_{i j}$ is the common interface area between connected blocks or nodes $i$ and $j$ (Figure 1); and $D_{i}$ is the distance from the center of block $i$ to the interface between blocks $i$ and $j$ (Figure 1). The flow potential term in Equation (13) is defined as

$\psi_{\beta \mathrm{i}}=\mathrm{P}_{\beta \mathrm{i}}-\rho_{\beta, \mathrm{ij}+1 / 2} \mathrm{~g} \mathrm{Z}_{\mathrm{i}}$ 
where $Z_{i}$ is the depth to the center of block $i$ from a reference datum.

For mass component transport, the flow term, or the net mass flux by advection and hydrodynamic dispersion of a component along the connection of nodes $i$ and $j$, is determined by

flow $_{\mathrm{ij}}^{\mathrm{k}}=\mathrm{F}_{\mathrm{A}, \mathrm{j}}^{\mathrm{k}}+\mathrm{F}_{\mathrm{D}, \mathrm{ij}}^{\mathrm{k}} \quad\left(\mathrm{k}=1,2,3, \ldots, \mathrm{N}_{\mathrm{c}}\right)$

where $\mathrm{F}_{\mathrm{A}, \mathrm{ij}}^{\mathrm{k}}$ and $\mathrm{F}_{\mathrm{D}, \mathrm{j}}^{\mathrm{k}}$ are the net mass fluxes by advection and hydrodynamic dispersion along the connection, respectively, with

$\mathrm{F}_{\mathrm{A}, \mathrm{ij}}^{\mathrm{k}}=\mathrm{A}_{\mathrm{ij}} \sum_{\beta}\left(\mathrm{X}_{\beta}^{\mathrm{k}}\right)_{\mathrm{ij}+1 / 2} \mathrm{~F}_{\beta, \mathrm{ij}}$

and

$\mathrm{F}_{\mathrm{D}, \mathrm{ij}}^{\mathrm{k}}=-\mathbf{n}_{\mathrm{ij}} \cdot \mathrm{A}_{\mathrm{ij}} \sum_{\beta} \underline{D}_{\beta}^{\mathrm{k}} \bullet \nabla\left(\rho_{\beta} \mathrm{X}_{\beta}^{\mathrm{k}}\right)$

where $\mathbf{n}_{\mathrm{ij}}$ is the unit vector along the connection of the two blocks $\mathrm{i}$ and $\mathrm{j}$.

The total heat flux along the connection of nodes $\mathrm{i}$ and $\mathrm{j}$, including advective, diffusive, conductive and radiation terms, may be evaluated, when using a finite-difference scheme, by

flow $_{\mathrm{ij}}^{\mathrm{N}_{\mathrm{j}}+1}=\sum_{\beta}\left[\left(\mathrm{h}_{\beta}\right)_{\mathrm{ij}+1 / 2} \mathrm{~F}_{\beta, \mathrm{j}}\right]+\sum_{\beta} \sum_{\mathrm{k}}\left\{\left(\mathrm{h}_{\beta}^{\mathrm{k}}\right)_{\mathrm{ij}+1 / 2} \mathrm{~F}_{\mathrm{D}, \mathrm{ij}}^{\mathrm{k}}\right\}+\mathrm{A}_{\mathrm{ij}}\left(\mathrm{K}_{\mathrm{T}}\right)_{\mathrm{ij}+1 / 2}\left[\frac{\mathrm{T}_{\mathrm{j}}-\mathrm{T}_{\mathrm{i}}}{\mathrm{D}_{\mathrm{i}}+\mathrm{D}_{\mathrm{j}}}\right]+\mathrm{A}_{\mathrm{ij}} \sigma_{0} \varepsilon_{\mathrm{ij}+1 / 2}\left(\mathrm{~T}_{\mathrm{j}}^{4}-\mathrm{T}_{\mathrm{i}}^{4}\right)$

In evaluating the "flow" terms in the above equations, (13), (16) and (19), subscript $i j+1 / 2$ is used to denote a proper averaging or weighting of fluid flow, component transport, or heat transfer properties at the interface or along the connection between two blocks or nodes $i$ and $\mathrm{j}$. The convention for the signs of flow terms is that flow from node $\mathrm{j}$ into node $\mathrm{i}$ is defined as "+" (positive) in calculating the flow terms. Wu and Pruess [18] present a general approach to calculating these flow terms associated with advective and dipersive mass transport and heat transfer in a multiphase system, using an irregular and unstructured, multidimensional grid.

The mass or energy sink/source in Equation (12) at node $\mathrm{i}, \mathrm{Q}_{\mathrm{i}}^{\mathrm{k}}$, is defined as the mass or energy exchange rate per unit volume of rocks or soils. It is normally used to treat boundary conditions, such as surface infiltration, pumping, and injection through wells.

Note that we present explicit, discrete expressions for estimating all the flow terms above, except for dispersive fluxes in Equation (18). This is because of the numerical difficulties introduced in handling the hydrodynamic tensor of dispersion, which is treated very differently with different numerical approaches, such as finite difference or finite element. In most formulations for solute transport, the off-diagonal terms and contributions of the dispersion tensor are ignored, and dispersive transport is considered only along the principal 
directions. However, a general procedure for using the integral finite difference to incorporate a full dispersion tensor is presented by $\mathrm{Wu}$ and Pruess [18].

Note that Equation (12) presents a precise form of the balance equation for each mass component and heat in a discrete form. It states that the rate of change in mass or energy accumulation (plus decay/generation, if existing) at a node over a time step is exactly balanced by inflow/outflow of mass and energy and also by sink/source terms, when existing for the node. As long as all flow terms have flow from node $i$ to node $j$ equal to and opposite to that of node $\mathrm{j}$ to node $\mathrm{i}$ for fluids, components, and heat, no mass or energy will be lost or created in the formulation during the solution. Therefore, the discretization in (12) is conservative.

\subsection{Numerical Solution Scheme}

There are a number of numerical solution techniques that have been developed in the literature over the past few decades to solve the nonlinear, discrete equations of reservoir simulations. When handling multiphase flow, multicomponent transport, and heat transfer in a multiphase flow system, the predominant approach is to use a fully implicit scheme. This is due to the extremely high nonlinearity inherent in those discrete equations and the many numerical schemes with different level of explicitness that fail to converge in practice. In this section, we discuss a general procedure to solve the discrete nonlinear Equation (12) fully implicitly, using a Newton iteration method.

Let us write the discrete non-linear Equation (12) in a residual form as

$$
\begin{aligned}
& \mathrm{R}_{\mathrm{i}}^{\mathrm{k}, \mathrm{n}+1}=\left\{\mathrm{A}_{\mathrm{i}}^{\mathrm{k}, \mathrm{n}+1}+\mathrm{G}_{\mathrm{i}}^{\mathrm{k}, \mathrm{n}+1}-\mathrm{A}_{\mathrm{i}}^{\mathrm{k}, \mathrm{n}}\right\} \frac{\mathrm{V}_{\mathrm{i}}}{\Delta \mathrm{t}}-\sum_{\mathrm{j} \in \eta_{\mathrm{i}}} \text { flow }_{\mathrm{ij}}^{\mathrm{k}, \mathrm{n}+1}-\mathrm{Q}_{\mathrm{i}}^{\mathrm{k}, \mathrm{n}+1}=0 \\
& \left.\mathrm{k}=1,2,3, \ldots, \mathrm{N}_{\mathrm{c}}+1 ; \mathrm{i}=1,2,3, \ldots, \mathrm{N}\right) .
\end{aligned}
$$

Equation (20) defines a set of $\left(\mathrm{N}_{\mathrm{c}}+1\right) \times \mathrm{N}$ coupled nonlinear equations that need to be solved for every balance equation of mass components and heat, respectively. In general, $\left(\mathrm{N}_{\mathrm{c}}+1\right)$ primary variables per node are needed to use the Newton iteration for the associated $\left(\mathrm{N}_{\mathrm{c}}+1\right)$ equations per node. The primary variables are usually selected among fluid pressures, fluid saturations, mass (mole) fractions of components in fluids, and temperatures. In many applications, however, primary variables cannot be fixed and must be allowed to vary dynamically in order to deal with phase appearance and disappearance [6,5]. The rest of the dependent variables, such as relative permeability, capillary pressures, viscosity and densities, partitioning coefficients, specific enthalpies, thermal conductivities, dispersion tensor, etc., as well as nonselected pressures, saturations, and mass (mole) fractions, are treated as secondary variables.

In terms of the primary variables, the residual equation (20) at a node $\mathrm{i}$ is regarded as a function of the primary variables at not only node I, but also at all its direct neighboring nodes j. The Newton iteration scheme gives rise to

$$
\sum_{\mathrm{m}} \frac{\partial \mathrm{R}_{\mathrm{i}}^{\mathrm{k}, \mathrm{n}+1}\left(\mathrm{x}_{\mathrm{m}, \mathrm{p}}\right)}{\partial \mathrm{x}_{\mathrm{m}}}\left(\delta \mathrm{x}_{\mathrm{m}, \mathrm{p}+1}\right)=-\mathrm{R}_{\mathrm{i}}^{\mathrm{k}, \mathrm{n}+1}\left(\mathrm{x}_{\mathrm{m}, \mathrm{p}}\right)
$$


where $\mathrm{x}_{\mathrm{m}}$ is the primary variable $\mathrm{m}$ with $\mathrm{m}=1,2,3, \ldots, \mathrm{N}_{\mathrm{c}}+1$, respectively, at node $\mathrm{i}$ and all its direct neighbors; $\mathrm{p}$ is the iteration level; and $\mathrm{i}=1,2,3, \ldots, \mathrm{N}$. The primary variables in (21) need to be updated after each iteration:

$\mathrm{x}_{\mathrm{m}, \mathrm{p}+1}=\mathrm{x}_{\mathrm{m}, \mathrm{p}}+\delta \mathrm{x}_{\mathrm{m}, \mathrm{p}+1}$

The Newton iteration process continues until the residuals $\mathrm{R}_{\mathrm{n}}^{\mathrm{k}, \mathrm{n}+1}$ or changes in the primary variables $\delta \mathrm{x}_{\mathrm{m}, \mathrm{p}+1}$ over an iteration are reduced below preset convergence tolerances.

Numerical methods are generally used to construct the Jacobian matrix for Equation (21), as outlined in Forsyth et al. [4]. At each Newton iteration, Equation (21) represents a system of $\left(\mathrm{N}_{\mathrm{c}}+1\right) \times \mathrm{N}$ linearized algebraic equations with sparse matrices, which are solved by a linear equation solver.

\subsection{Treatment of Initial and Boundary Conditions}

A set of initial conditions is required to start a transient simulation, i.e., a complete set of primary variables need to be specified for every gridblock or node. A commonly used procedure for specifying initial conditions is the restart option, in which a complete set of initial conditions or primary unknowns is generated in a previous simulation with proper boundary conditions described.

Because of more physical and chemical constraints, boundary conditions for a multiphase flow and transport problem are generally much more difficult to handle than for a singlephase situation. When using a block-centered grid, first-type or Dirichlet boundary conditions, can be effectively treated with the "inactive cell" or "big-volume" method, as normally used in the TOUGH2 code [13]. In this method, a constant pressure/saturation/concentration/temperature node is specified as an inactive cell or with a huge volume, while keeping all the other geometric properties of the mesh unchanged.

With finite-element or edge-centered finite-difference grids, first-type boundary conditions and Neuman boundary conditions can be treated using a generalized, sink/source term approach $[17,20]$. Certain flux-type boundary conditions are easy to handle for a situation where flux distribution along the boundary is known, such as in dealing with surface infiltration. However, a description of more general types of flux- or mixed-boundaries, such as seepage faces and multilayered wells, is part of the solution, and general procedures of handling such boundary conditions are discussed in [19].

\subsection{Fractured Media}

The mathematical and numerical formulations discussed above are applicable to both single-continuum and multi-continuum media, as long as the physical processes concerned can be described in a continuum sense within either continuum. When handling flow and transport through a fractured rock using the numerical formation of this section, fractured media (including explicit fracture, dual, or multiple continuum models) can be considered as special cases of unstructured grids [13]. Then, a large portion of the work consists of generating a mesh that represents both the fracture system and the matrix system under consideration. Several fracture and matrix subgridding schemes exist for designing different meshes for different fracture-matrix conceptual models [14]. 
Once a proper grid of a fracture-matrix system is generated, fracture and matrix blocks are identified to represent fracture and matrix domains, separately. Formally they are treated identically for the solution in the model. However, physically consistent fracture and matrix properties, parameter weighting schemes, and modeling conditions must be appropriately specified for both fracture and matrix systems.

\section{APPLICATION EXAMPLES}

The unified mathematical and numerical formulations discussed in Sections 3 and 4, cover most commonly encountered applications of reservoir simulation and groundwater modeling. These model formulations have been tested using a multiphase flow code [19] and a nonisothermal flow and transport code [18] to demonstrate their capability. Tested results included the following simulation scenarios:

(1) Single-phase liquid

(2) Single-phase gas flow

(3) Unsaturated liquid flow using Richards' equation

(4) Two-phase gas and liquid flow

(5) Three-phase flow

(6) Density-dependent solute transport in single liquid phase systems

(7) Tracer transport in isothermal and nonisothermal multiphase systems

(8) Heat transfer in multiphase systems

In addition, it can be shown that the model formations can also be used to simulate heat conduction in solids and diffusion in solids or in a stagnated fluid, which are treated as special cases of these proposed formations.

\section{SUMMARY AND CONCLUDING REMARKS}

A unified numerical formulation has been discussed for modeling fluid flow, mass transport, and heat-transfer processes through porous and fractured media. This work takes advantage of the fact that governing equations used for describing various flow and transport phenomena in porous media are all generally based on the same form of mass and/or energy conservation laws. This indicates that there may exist a unified formulation and numerical scheme applicable to all of these physical processes. This paper explores such a possibility by proposing a generalized framework as well as mathematical formulation for modeling all known transport phenomena in porous media. The numerical implementation of the unified formulation is based on a control-volume spatial discretization with an unstructured grid and time discretization with a fully implicit finite-difference method. The final discrete linear or nonlinear equations are handled fully implicitly, using Newton iteration.

As demonstrated in this paper, this unified approach makes it possible to model a particular process of porous-medium flow and transport becomes simply a matter of defining a set of state variables, along with evaluation of their interrelations or mutual effects. 


\section{ACKNOWLEDGMENTS}

The author would like to thank Guoxiang Zhang and Dan Hawkes for their review of this paper. This work was supported in part by the Assistant Secretary for Energy Efficiency and Renewable Energy, Office of Geothermal Technologies of the U.S. Department of Energy under Contract No. DE-AC03-76SF00098.

\section{REFERENCES}

1. K. Aziz, K. and A. Settari, Applied Science Publishers LTD, London, 1979.

2. J. Bear, American Elsevier Publishing Company, New York, 1972.

3. K. H. Coats, Society of Petroleum Engineers, Richardson, Texas, pp.48-1-48-20, 1987.

4. P. A. Forsyth, Y. S. Wu, and K. Pruess, Advances in Water Resources, Vol. 18, pp. 2538, 1995.

5. P. A. Forsyth, International Journal for Numerical Methods in Fluids, Vol. 19, pp.10551081, 1994.

6. $\quad$ P. A. Forsyth, Advances in Water Resources, Vol. 16, pp. 351-370, 1993.

7. $\quad$ P. A. Forsyth, SIAM J. Sci. Statist. Comput. 12(5), pp.1029-1057, 1991.

8. P. S. Huyakorn, S. Panday, and Y. S. Wu, Journal of Contaminant Hydrology, 16, pp. 109-130, 1994.

9. $\quad$ P. S. Huyakorn and G. F. Pinder, Academic Press, Inc., New York, 1983.

10. J. Istok, American Geophysical Union Water Resources Monograph 13, 1989.

11. T. N. Narasimhan and P. A. Witherspoon, Water Resources Research, 12(1), pp. 57-64, 1976.

12. D. W. Peaceman, Developments in Petroleum Sciences, 6, Elsevier Scientific Publishing Company, Amsterdam-Oxford-New York, 1977.

13. K. Pruess, Report LBL-29400, Lawrence Berkeley National Laboratory, Berkeley, California, 1991.

14. K. Pruess, Report LBL-15227, Berkeley, California: Lawrence Berkeley National Laboratory, 1983.

15. A. E. Scheidegger, Third Edition, University of Toronto Press, Toronto, 1974.

16. A. E. Scheidegger, J. Geophys. Res., Vol. 66, pp. 3273-3278, 1961.

17. G. W. Thomas, G. W., International Human Resources Development Corporation, Boston, 1982.

18. Y. S. Wu and K. Pruess, Advance in Water Resources, Vol. 23, pp. 699-723, 2000.

19. Y. S. Wu, Water Resources Research, Vol. 36, N0. 3, pp. 807-814, 2000.

20. Y. S. Wu, P. A. Forsyth, and H. Jiang, Journal of Contaminant Hydrology, 23, pp. 157$184,1996$. 